\title{
« Consommation collaborative » ou la rencontre improbable entre organisations, communautés et individus
}

\author{
Myriam Ertz ${ }^{\mathrm{a}}$
}

\begin{abstract}
Tout est vague à un point que vous n’imaginez même pas, jusqu'au moment où vous avez essayé de rendre cette chose précise. (Bertrand Russell, auteur, mathématicien et philosophe britannique [1872-1970])
\end{abstract}

RÉSUMÉ. Malgré la multiplication des études empiriques sur le sujet de la consommation collaborative, force est de constater que les bases conceptuelles et théoriques relatives à ce phénomène demeurent relativement faibles. Cette constatation est d'autant plus remarquable eu égard au fait que la jonction entre individus, organisations et communauté, qu'induit la consommation collaborative, a des répercussions profondes sur les circuits marchands conventionnels. Cet article vise à étoffer les fondements théoriques et conceptuels liés au concept de consommation collaborative en l'explorant sous l'angle plus circonscrit des pratiques de mutualisation et de redistribution de biens tangibles, appelées pratiques de seconde vie des objets. L'étude propose ainsi, tout d'abord, une définition de la consommation collaborative, suivie ensuite par l'introduction d'un cadre conceptuel dans lequel s'inscrit le sous-ensemble des pratiques de seconde vie des objets, avant de conclure sur l'impact du phénomène sur le processus du consommateur.

\begin{abstract}
Despite the multiplication of empirical studies on the topic of collaborative consumption, the conceptual and theoretical bases pertaining to this phenomenon remain rather weak. This observation is even more remarkable considering the fact that the junction between individuals, organizations, and community, entailed by collaborative consumption, has strong repercussions on conventional trading circuits. This article aims at developing the conceptual and theoretical foundations related to the concept of collaborative consumption by exploring the phenomenon under the narrower angle of product second life practices. Therefore, the study proposes, first, a definition of the term collaborative consumption, then followed by the introduction of a conceptual framework in which are anchored the subset of product second life practices. The article concludes with the impact of the phenomenon on the consumer process.
\end{abstract}

\section{Définition de la consommation collaborative}

État des lieux et problématique

La consommation collaborative (CC) est un terme bien vaste et souvent rattaché à un ensemble de concepts nébuleux et abstraits tels que «l'économie du partage » (sharing economy), l'« économie des pairs » (peer economy), l'« économie gig » (gig economy), l'« économie de plateformes » (platform economy), l'« économie des applications » (app economy), la « consommation

\footnotetext{
a Professeure, Ph. D., Adm.A., Université du Québec à Chicoutimi
}

pair-à-pair » (peer-to-peer consumption) (Botsman, 2013; Belk, 2014) ou encore le "capitalisme basé sur la foule » (crowd-based capitalism) (Sundararajan, 2016). D’après Botsman (2013, 2015a, 2015b), le concept de consommation collaborative ne serait qu'une composante de la notion plus large d'« économie collaborative »(Collaborative Economy), laquelle est définie comme :

Une économie construite sur la base de réseaux d'individus et de communautés connectés par des institutions centralisées, transformant la 
manière dont nous pouvons produire, consommer, financer, et nous éduquer. (Botsman, 2015a, p. 1, [traduction libre]).

D'après Botsman (2013), l'économie collaborative se subdivise en quatre grands domaines, dont la production collaborative (ex. les Fablab), l'éducation collaborative (ex. la Khan Academy), la finance collaborative (ex. Kiva) et, la consommation collaborative. Lorsque la consommation collaborative est fortement axée sur les technologies, il s'agit de l'économie de partage (ex. Airbnb), et lorsqu'elle est moins fortement axée sur la technologie, il s'agit d'une consommation ancrée sur le territoire (ex. Accorderies).

Cette classification comprend toutefois de nombreux points discutables. Par exemple, les services financiers sont des biens de consommation largement étudiés dans le comportement du consommateur, et constituent classiquement une forme de consommation (Zhu et al., 2012). De même pour l'éducation (Solomon, Zaichkowksy et Polegato, 2011). Seule la production serait distincte, mais là encore, les travaux récents sur le «travail de l'ombre » (shadow work) qu'effectuent les consommateurs dans leurs activités de consommation (p. ex. : utiliser un guichet bancaire automatique, faire le plein d'essence) (Lambert, 2015) ou la "prosomption» (prosumption) (Ritzer, 2015), suggèrent plutôt que production et consommation ont de plus en plus tendance à se réaliser de manière conjointe, comme le démontre le «libre-service »(Lambert, 2015), la " coproduction » (Prahalad et Ramaswamy, 2000), ou la «personnalisation de masse »(Kaplan et Haenlein, 2006). Botsman (2015b), ellemême, rattache fortement certains pans de la production collaborative à la consommation collaborative, car réalisés de pair-à-pair. Par ailleurs, la notion d'« économie du partage » a été à plusieurs reprises critiquée, car utilisée pour décrire des pratiques qui sont aux antipodes de l'échange désintéressé et intégrateur, induit par la notion de partage (Slee, 2015; Eckhardt et Bardhi, 2015; Schor, 2015; John, 2013). Du fait des contraintes d'espaces de l'article, cette notion ne sera pas traitée ici.

Malgré ces écueils, et devant le vide conceptuel béant sur le sujet, la conceptualisation de Botsman et Rogers (2010) et Botsman (2013, 2015a, 2015b) a toutefois été reprise, et occasionnellement adaptée, par les grands think tanks et groupes de pression promouvant la consommation collaborative tels que OuiShare, Shareable, Wired, The Collaborative Centre on Sustainable Consumption and Production, Vision Capitalist, WebStrategist, Collaboriamo, ou Collabortiveconsumption.com; mais aussi par des organisations gouvernementales (Pôle interministériel de prospective et d'anticipation des mutations économiques [PIPAME] en France), institutionnelles (University of California Transportation Center [UCTC]) ou privées (PricewaterhouseCooper, Nielsen, Altimeter, Vision Critical, Crowd Companies, Forbes, The Economist), lesquels ont tous, en raison de leur influence, contribué à la popularisation de cette vision de la consommation collaborative en tant que composante de l'économie collaborative. Malgré les nombreux travaux et développements sur le sujet, une définition scientifique claire de la CC demeure toutefois manquante comme l'a souligné Rachel Botsman, en personne, à travers un article intitulé ironiquement "The Sharing Economy Lacks a Shared Definition » (Botsman, 2013). Outre le glissement sémantique du terme de «consommation collaborative » au profit de celui d'«économie du partage ", qui n'arrange en rien les choses, l'article offre peu de nouveautés en matière d'assises conceptuelles. Ce n'est pas non plus la vocation première d'un article journalistique. Par conséquent, le besoin d'une définition scientifique adéquate demeure vivace.

\section{Retour aux origines}

Le concept de « consommation collaborative » a initialement été introduit par Felson et Spaeth (1978), bien avant le développement d'Internet, des applications mobiles et des réseaux sociaux, dans une perspective bien différente de celle attribuée aujourd'hui au concept. Dans leur article "Community structure and collaborative consumption : A routine activity approach ", paru dans la revue American Behavioral Scientist, les deux auteurs américains ont basé leur recherche sur les fondements théoriques de l'écologie humaine de Hawley (1950), afin de décrire la consommation collaborative comme des événements où une ou plusieurs personnes consomment des biens ou des services économiques en engageant des activités avec une (ou plusieurs) autre(s) personne(s). Ces travaux ont amené à une définition très large 
de la CC comme faisant référence à des événements requérant une forte cooccurrence spatiotemporelle et devant être analysés sous l'angle de la coordination et de la concurrence humaine. Les activités collaboratives peuvent être réalisées en contact direct avec des individus ou non (Felson et Spaeth, 1978). De plus, l'unité d'analyse devrait également se situer au niveau des « consommateurs » (Felson et Spaeth, 1978). Si ce niveau d'analyse s'accorde bien avec la discipline du comportement du consommateur ainsi qu'avec sa mission (Bazerman, 2001), la conceptualisation initiale proposée par Felson et Spaeth (1978) est toutefois trop large et peut inclure des activités aussi triviales que l'utilisation d'une laveuse pour la lessive familiale ou le partage d'un repas entre amis.

De plus, le contexte macro-environnemental de la fin des années 1970 caractérisant la rédaction de cet article est très différent de celui qui s'applique au cours de la seconde décennie du XXIe siècle. Du temps de Felson et Spaeth (1978), c'était le téléphone qui constituait une technologie aux implications déterminantes pour les entreprises et les consommateurs. Internet et les réseaux sociaux ont depuis largement pris le relais. Cette évolution technologique à elle seule donne une dimension tout autre à la consommation collaborative, car elle permet des échanges de tous types de ressources à une échelle inégalée du temps des deux auteurs.

\section{Développements récents}

Des définitions plus récentes ont ainsi été élaborées afin de caractériser le phénomène de la CC actuelle, dans le contexte des sciences de gestion et au-delà. Toutefois, certaines d'entre elles, pour ne pas dire la plupart, demeurent relativement insatisfaisantes. Tout d'abord, la CC a essentiellement été considérée comme un phénomène purement en lien avec le Web (Belk, 2014; Hamari et al., 2015). La définition de Gansky (2010) du concept de "Mesh », un dédoublement technologique du concept de CC de Botsman et Rogers (2010), en témoigne notamment. Elle définit "The Mesh ", comme un vieux concept faisant référence aux ouvertures entre les mailles d'un filet, et qui se rapporterait donc à « un type de réseau permettant à tout nœud [node] d'être relié dans n'importe quelle direction avec n'importe quel autre nœud dans un système » (Gansky, 2010, p. 17, [traduction libre]).

La coopération induite par les technologies Web (p. ex. : file-sharing [Giesler, 2006]) a certes formé la matrice des plateformes d'échange facilitées par le Web (Botsman et Rogers, 2010; Gansky, 2010; Airgrain, 2012; Nissanoff, 2006), et a redonné un nouveau souffle à des pratiques considérées comme épiphénoménales à la société de consommation (Botsman et Rogers, 2010; Ritzer, 2014, 2015). Toutefois, les pratiques hors ligne classiques (p. ex. : marché de l'occasion, troc) ne sont pas moins collaboratives que leurs équivalences technocentrées, et ce faisant, posent la question de la validité de leur exclusion de la CC.

De plus, la CC regroupe également des pratiques basées sur la location à titre gratuit ou payant, appelées "Accès » (Access) (Bardhi et Eckhardt, 2012), ou plus largement Systèmes de produits services (Product Service Systems [PSS]) (Tukker, 2004; Mont, 2002, 2004; Leismann et al., 2013; Fisk, 1973; Ostrom et Hess, 2007; Varian, 2000). Toutefois, la littérature managériale, voire scientifique (Lamberton et Rose, 2012; Belk, 2014), a par trop souvent limité la notion de CC à celle d'Accès, malgré le fait que les pratiques impliquant un transfert de propriété induisent généralement aussi des échanges pair-à-pair et donc collaboratifs, car tout échange avec un humain demande un degré de collaboration quelconque (Hermann et Soiffer, 1984; Bardhi et Arnould, 2005; Sherry, 1990; Belk et al., 1988; Gregson et Crewe, 2003; Stone et al., 1996; Guiot et Roux, 2010).

Certaines conceptualisations excluent également l'échange gratuit du spectre de la consommation collaborative (Belk, 2014). Toutefois, le monde du don a été de manière récurrente identifié comme étant vecteur de socialité, de réciprocité et donc, de collaborativité (Belk et Coon, 1993; Arnould et Rose, 2016; Bajde, 2013; Appadurai, 1986). Son exclusion amputerait donc la CC d'une de ses composantes les plus humaines et les plus importantes, notamment dans les pratiques de délaissement d'objets (Kijiji, 2015, 2016, 2017). 
La plupart des études managériales ainsi que de nombreux travaux scientifiques considèrent que les ressources d'entreprises privées mises à la disposition des consommateurs constituent une des facettes de la CC. Ainsi, Zipcar (Bardhi et Eckhardt, 2012), Bixi (Lamberton et Rose, 2012), ou encore Car2Go (Matzler et al., 2015) ont été identifiées comme des formes de CC. Or, il demeure assez difficile de distinguer en quoi ces entreprises diffèrent des entreprises de location classiques telles qu'Avis, Hertz ou Enterprise. Et dans le cas où elles n'en diffèrent pas, les entreprises de location conventionnelles susmentionnées favoriseraient-elles le partage? Feraient-elles en réalité partie de l'« économie du partage » à leur insu? Si ces considérations peuvent prêter à sourire, elles témoignent de l'incohérence conceptuelle régnant au sein de la recherche scientifique sur le sujet, complexifiant d'autant plus la tâche de classifier les modèles d'affaires émergents et les formes de consommation alternatives.

Dans le but de mieux conceptualiser la consommation collaborative, cet article se base sur les développements théoriques récents sur le sujet (Ertz et al., 2016a, 2016b) et réconciliant les différents questionnements soulevés précédemment. Selon ces travaux, la CC :

(1) Se décline en ligne, mais aussi hors ligne (Botsman et Rogers, 2010; Ertz et al., 2016a).

(2) Implique des échanges de redistribution avec transfert de droit de propriété (ex. don), mais aussi des échanges de mutualisation sans transfert des droits de propriété (ex. location temporaire) (Botsman et Rogers, 2010; Ertz et al., 2016a; Gansky, 2010; Belk, 2014; Hamari et al., 2015).

(3) Comprend les échanges payants, de troc, mais aussi les échanges gratuits (Botsman et Rogers, 2010; Ertz et al., 2016a; Gansky, 2010; Hamari et al., 2015).

(4) Exclut les modèles d'affaires impliquant des ressources détenues et commercialisées par des entreprises privées (ex. compagnies de taxis, hôtels) (Ertz et al., 2016a).
Plusieurs aspects de cette conceptualisation sont une reprise des définitions initiales de Botsman et Rogers (2010), ou de Gansky (2010). La différence principale réside dans l'exclusion des systèmes d'échanges impliquant des ressources organisationnelles. Cette vision comporte des implications majeures puisqu'elle diffère ainsi complètement de nombreux travaux universitaires sur le sujet (Lamberton et Rose, 2012; Bardhi et Eckhardt, 2012; Parkes et al., 2013; Matzler et al., 2015; Ciari et al., 2013). La CC est ainsi définie comme :

L'ensemble des systèmes de circulation de ressources permettant aux consommateurs d'utiliser et de fournir de manière temporaire ou permanente des ressources de valeur à travers une interaction directe avec un autre consommateur ou à travers un médiateur (Ertz et al., 2016b, p. 6, [traduction libre]).

L'affinage conceptuel récent de Ertz et Durif (2017), au sujet des intermédiaires pouvant participer à un échange, amène à remplacer le terme de médiateur par celui d'intermédiaire et à amplifier le fait que les ressources proviennent bien d'autres consommateurs. Un médiateur prend possession d'une ressource et la redistribue de manière temporaire ou permanente en déterminant les termes et les conditions de l'échange. Un intermédiaire, en revanche, ne prend pas possession de la ressource, mais facilite simplement la mise en relation d'échangeurs potentiels, et intervient peu ou pas du tout dans les termes et les conditions de l'échange. La CC est donc définie comme :

L'ensemble des systèmes de circulation de ressources permettant aux consommateurs d'utiliser et de fournir de manière temporaire ou permanente des ressources de valeur à travers une interaction directe avec un autre consommateur ou à travers un intermédiaire.

Une explication plus précise des fondements théoriques de cette définition et, par conséquent, de la manière dont elle diffère de la définition originale de Botsman et Rogers (2010), est développée dans la partie suivante. 


\section{Cadre conceptuel de la consommation collaborative et de la seconde vie des objets}

Les divergences conceptuelles esquissées précédemment démontrent bien le vide théorique béant entourant la CC et la nécessité d'y remédier, comme cela sera élaboré dans cette étude. À première vue, il apparaît peu évident d'identifier ce qui fait qu'une pratique est « collaborative » ou non. Est-ce le fait qu'elle soit réalisée de pair-àpair? Quid alors des achats d'occasion dans une boutique de seconde main? Est-ce alors plutôt le fait qu'elle soit réalisée de manière informelle, ou passant sous le radar des autorités? Quid alors des reventes privées organisées par le gouvernement notamment pour les voitures? Serait-ce encore le fait qu'elle implique uniquement l'accès aux biens? Quid alors de toutes ces pratiques qui permettent d'étendre la vie d'un objet par le transfert de sa propriété?

Cet article utilise la théorie des économies hybrides (Scaraboto, 2015; Perret, 2015; Corciolani et Dalli, 2014; Dalli et Corciolani, 2008; Robert et al., 2014) pour développer une conceptualisation plus approfondie de la CC et ce faisant, de la Seconde Vie des Objets (SVO). Cette approche théorique démontre comment la montée en puissance des échanges informels entre individus, soutenus par le Web, change le rôle traditionnel des intervenants de l'économie, tels que les "consommateurs », et les "intermédiaires ». Le paradigme économique et marketing classique distingue clairement l'organisation/la production, d'un côté, et la société/consommation, de l'autre (Perret, 2015; Robert et al., 2014; Ritzer, 2015; Lambert, 2015). En somme, d'une part, on retrouve les organisations (p. ex. : entreprises, distributeurs, détaillants) et de l'autre, les consommateurs (Perret, 2015; Scaraboto, 2015). Les consommateurs achètent et consomment les biens que produisent et distribuent les organisations. La CC, en revanche, bouscule ce paradigme fondé sur une conceptualisation dichotomique (production ou consommation), moderniste, essentialiste et discrète.

Ainsi, pour analyser la CC, il convient plutôt d'utiliser les apports conceptuels récents qui suggèrent une vision anti-essentialiste et continue des échanges. Cette perspective implique plutôt de considérer l'économie comme un ensemble de systèmes de circulation de ressources (Arnould et Rose, 2016; Bajde, 2013). Dans ces systèmes, il y a « enchâssement " plutôt que « distinction » entre production et consommation (Corciolani et Dalli, 2014; Dalli et Corciolani, 2008; Perret, 2015; Laville, 2008; Lessig, 2008), d'où la notion d'économie bybride (Scaraboto, 2015; Arsel et Dobscha, 2011; Corciolani et Dalli, 2014), dans laquelle les échanges entre individus sont ramenés au premier plan, alors que les organisations font plutôt office d'intermédiaires (Tussyadiah, 2015; Airgrain, 2012; Nissanoff, 2006).

Le consommateur n'est plus uniquement acheteur-consommateur, mais intervient également dans la chaîne de production de valeur d'une organisation. Il produit et commercialise ses propres biens ou encore échange des biens préacquis directement avec d'autres consommateurs (Scaraboto, 2015; Perret, 2015; Corciolani et Dalli, 2014; Dalli et Corciolani, 2008; Arnould et Rose, 2016; Tussyadiah, 2015; Airgrain, 2012; Nissanoff, 2006; Robert et al., 2014). Ainsi, dans la CC, il est plus approprié de parler d'« utilisateur » que de « consommateur». De plus, l'individu est également "fournisseur », à travers la provision de ressources au sens large (CC) (Ertz et al., 2016b). La capacité des consommateurs d'endosser un double rôle d'utilisateur et de fournisseur constitue donc le critère permettant d'identifier si un système de circulation de ressource particulier, relève de la $\mathrm{CC}$ ou non. Ce n'est pas le cas des Car2Go, Bixi et autres Zipcar, malgré leurs vertus intrinsèques en matière de mobilité et de durabilité.

Le tableau 1 définit plus particulièrement les rôles d'utilisateur et de fournisseur. Un « intermédiaire facilitateur » constitue un facilitateur de l'échange qui n'intervient pas dans les modalités d'échange d'une ressource et ne prélève pas de commission sur cet échange (p. ex. : Kijiji). D'autres exemples incluent eBay ou la section des biens usagés d'Amazon, et à un niveau plus local, LesP $A C$ et les municipalités ou organismes communautaires organisant des brocantes ou des marchés aux puces. En revanche, un « intermédiaire médiateur » fait référence à une entité qui gère la relation d'échange d'une ressource entre un utilisateur et un fournisseur et en prélève une 
ressource. Par exemple, la plateforme AmigoExpress met en relation des utilisateurs souhaitant se déplacer et des fournisseurs qui offrent des solutions de transport à d'autres moyennant une somme d'argent. La plateforme se charge toutefois de l'ensemble du processus d'échange y compris de la mise en relation d'un utilisateur particulier avec un fournisseur spécifique en échange, des modalités d'échange en plus du prélèvement d'une contrepartie sur l'échange réalisé. D'autres exemples comprennent Uber ou Airbnb, et à un niveau plus local, Les Accorderies, Minitrade ou Renaissance, lesquels impliquent tous des échanges de ressources de consommateurs.

\begin{tabular}{|c|c|c|}
\hline & Utilisateur & Fournisseur \\
\hline Fonction & $\begin{array}{l}\text { Cherche à utiliser une ressource fournie soit } \\
\text { directement, par un autre consommateur (c.- } \\
\text { à.-d. le fournisseur), soit indirectement à } \\
\text { travers la médiation d'une organisation } \\
\text { comme un « intermédiaire facilitateur » ou un } \\
\text { « intermédiaire médiateur » (à but lucratif ou } \\
\text { non) }\end{array}$ & $\begin{array}{l}\text { Fournit une ressource spécifique soit } \\
\text { directement, à un consommateur (c.-à.- } \\
\text { d. l'utilisateur), soit indirectement à } \\
\text { travers un « intermédiaire facilitateur » } \\
\text { ou un « intermédiaire médiateur » (à but } \\
\text { lucratif ou non) }\end{array}$ \\
\hline \multirow[t]{2}{*}{ Action } & «Utilisation »: & «Provision »: \\
\hline & $\begin{array}{l}\text { - } \quad \text { Redistribution : achat de seconde } \\
\text { main (en incluant l'achat de produits } \\
\text { remis à neuf ou en état), réception de } \\
\text { don, troc; } \\
\text { Mutualisation : } \\
\text { location/emprunt/accès à une } \\
\text { ressource à titre gratuit ou pour une } \\
\text { compensation (en excluant l'accès } \\
\text { aux ressources provenant } \\
\text { uniquement d'entreprises) }\end{array}$ & $\begin{array}{l}\text { - } \quad \text { Redistribution : vente } \\
\text { d'occasion, don, troc (en } \\
\text { incluant les programmes de } \\
\text { reprise avec une organisation); } \\
\text { - Mutualisation : location/prêt } \\
\text { d'une ressource à titre gratuit } \\
\text { ou pour une compensation }\end{array}$ \\
\hline
\end{tabular}

Tableau 1 - Définition de l'utilisateur et du fournisseur Source : adapté de Van de Walle et al. (2012), et Ertz et al. (2016a, b).

À partir des notions d'intermédiaire (facilitateur et médiateur), la CC peut être mieux appréhendée selon la perspective plus large de «systèmes de circulation de ressources » empruntée à Arnould et Rose (2016). En accord avec une vision non-essentialiste (Arnould et Rose, 2016) de la CC, la collaboration n'est pas présente à des niveaux identiques à travers différents systèmes de circulation de ressources. Il y aurait plutôt différents niveaux d'intensité collaborative, lesquels peuvent toutefois se regrouper sous quatre configurations génériques, telles que présentées dans les colonnes 2 à 5 du tableau 2 . La dernière colonne démontre comment la consommation classique se distingue par rapport aux différents niveaux de CC. 


\begin{tabular}{|c|c|c|c|c|c|}
\hline & \multirow{2}{*}{$\begin{array}{l}\text { Collaboration } \\
\text { pure }\end{array}$} & \multirow{2}{*}{$\begin{array}{l}\text { Collaboration } \\
\text { facilitée }\end{array}$} & \multicolumn{2}{|c|}{ Collaboration de médiation } & \multirow{2}{*}{$\begin{array}{l}\text { Consommation } \\
\text { classique }\end{array}$} \\
\hline & & & $\begin{array}{l}\text { Collaboration } \\
\text { d'utilisation }\end{array}$ & $\begin{array}{l}\text { Collaboration de } \\
\text { délaissement }\end{array}$ & \\
\hline Processus & $\begin{array}{l}\text { Utilisateur et four- } \\
\text { nisseur sont des } \\
\text { consommateurs } \\
\text { qui échangent une } \\
\text { ressource }\end{array}$ & $\begin{array}{l}\text { L'acquéreur et le } \\
\text { délaisseur sont des } \\
\text { consommateurs } \\
\text { qui échangent une } \\
\text { ressource }\end{array}$ & $\begin{array}{l}\text { L'acquéreur uti- } \\
\text { lise une } \\
\text { ressource } \\
\text { fournie par le } \\
\text { fournisseur à tra- } \\
\text { vers un } \\
\text { médiateur }\end{array}$ & $\begin{array}{l}\text { Le fournisseur } \\
\text { fournit une } \\
\text { ressource à un } \\
\text { utilisateur par } \\
\text { l'entremise d'un } \\
\text { médiateur }\end{array}$ & $\begin{array}{l}\text { Le consommateur } \\
\text { achète un bien } \\
\text { commercialisé par } \\
\text { une entreprise }\end{array}$ \\
\hline $\begin{array}{l}\text { Exemple de } \\
\text { processus }\end{array}$ & $\begin{array}{l}\text { L'achat d'une télé- } \\
\text { vision d'occasion } \\
\text { lors d'une vente- } \\
\text { débarras }\end{array}$ & $\begin{array}{l}\text { L'achat d'une télé- } \\
\text { vision d'occasion } \\
\text { par l'entremise de } \\
\text { Kijiji }\end{array}$ & $\begin{array}{l}\text { L'achat d'une té- } \\
\text { lévision d'occa- } \\
\text { sion dans un ma- } \\
\text { gasin de seconde } \\
\text { main (ex. Friprix) }\end{array}$ & $\begin{array}{l}\text { La vente ou le } \\
\text { don d'une télévi- } \\
\text { sion d'occasion à } \\
\text { un magasin de } \\
\text { seconde main } \\
\text { (ex. Friprix) }\end{array}$ & $\begin{array}{l}\text { L'achat d'une } \\
\text { télévision neuve } \\
\text { chez Best Buy }\end{array}$ \\
\hline Type d'échange & $\mathrm{C} 2 \mathrm{C}$ & $\mathrm{C} 2 \mathrm{C}$ & $\mathrm{O} 2 \mathrm{C}$ & $\mathrm{C} 2 \mathrm{O}$ & $\mathrm{B} 2 \mathrm{C}$ \\
\hline $\begin{array}{l}\text { Rôle possible du } \\
\text { consommateur }\end{array}$ & $\begin{array}{l}\text { Utilisateur et } \\
\text { fournisseur }\end{array}$ & $\begin{array}{l}\text { Utilisateur et } \\
\text { fournisseur }\end{array}$ & Utilisateur & Fournisseur & $\begin{array}{l}\text { Acheteur- } \\
\text { consommateur }\end{array}$ \\
\hline $\begin{array}{l}\text { Présence d'un } \\
\text { intermédiaire } \\
\text { facilitateur }\end{array}$ & Non & Oui & Non & Non & Non \\
\hline $\begin{array}{l}\text { Présence d'un } \\
\text { intermédiaire } \\
\text { médiateur }\end{array}$ & Non & Non & Oui & Oui & Non \\
\hline
\end{tabular}

Note $: \mathrm{C} 2 \mathrm{C}=$ Consommateur à consommateur; $\mathrm{O} 2 \mathrm{C}=$ Organisation à consommateur; $\mathrm{C} 2 \mathrm{O}=$ Consommateur à organisation; $\mathrm{B} 2 \mathrm{C}=$ Entreprise à consommateur.

Tableau 2-Niveaux d'intensité collaborative

Source : adapté de Ertz et al. (2016a, 2016b).

Collaboration pure. Fait référence aux échanges pair-à-pair dans lesquels les individus échangent directement une ressource spécifique, sans aucun intermédiaire, qu'il soit facilitateur ou médiateur. Les exemples incluent les marchés aux puces, brocantes, ventes-débarras et autres événements ponctuels ou réguliers permettant des échanges directs entre consommateurs.

Collaboration facilitée. Indique des échanges pair-àpair dans lesquels les individus peuvent échanger une ressource spécifique par l'entremise d'un intermédiaire facilitateur, typiquement un site Internet, ou encore les divers organismes communautaires ou municipaux, permettant de mettre en relation l'acquéreur et le délaisseur.

Collaboration d'utilisation. Cette perspective fait référence à l'utilisation d'une ressource rendue disponible par un médiateur. La ressource en question a toutefois été fournie par un individu fournisseur auprès du médiateur. Par exemple, 
les produits neufs tels que les peintures ou les produits de beauté neufs commercialisés au sein des centres de don Renaissance impliquent un niveau de collaboration nulle puisqu'ils sont neufs, approvisionnés par des entreprises, et n'ont donc pas été fournis par des individus. En revanche, l'achat d'un ordinateur remis à neuf chez Best Buy constitue une forme de collaboration d'acquisition puisque le bien a dû initialement être délaissé par un individu fournisseur auprès de l'intermédiaire médiateur Best Buy avant d'être remis à neuf et revendu à un quelconque utilisateur.

Collaboration de provision. Le corollaire de la collaboration d'utilisation. Il s'agit de la provision d'une ressource auprès d'un intermédiaire médiateur, lequel redistribuera la ressource en question à des utilisateurs potentiels. Un consommateur pourrait donner ou revendre un produit à un magasin de seconde main ou encore rapporter un produit électronique utilisé chez Best Buy, pour être revendu à d'autres consommateurs (produits reconditionnés).

Consommation classique. Il s'agit de la consommation classique - y compris locale, éthique, verte, citoyenne, écologique, équitable, responsable ou autre - au cœur de la littérature marketing. Un consommateur achète un bien neuf avec de l'argent auprès d'un commerçant.

La prochaine section identifie plus en détail le processus du consommateur sous-jacent à ces formes de collaboration.

\section{Le processus du consommateur}

\section{Consommation conventionnelle et "délégation 》}

La « collaboration pure » (C2C) est l'exact opposé de la « consommation conventionnelle » (B2C). Toutefois, force est de constater que dans les échanges collaboratifs interviennent souvent de nombreux intermédiaires censés faciliter les échanges, tels que des plateformes Web (ex. Kijiji) ou des applications mobiles (ex. Uber), entres autres. La perspective dichotomique entreprise versus consommateur est donc peu appropriée à l'examen de la multiplicité des configurations d'échanges possibles dans la consommation collaborative (CC). Il importe donc d'adopter une perspective mieux adaptée à ces échanges, afin de comprendre le processus du consommateur dans les divers cas possibles.

Selon des apports théoriques récents, le marché ne serait rien d'autre qu'un " réseau dense de mécanismes et d'opérations, permettant aux acteurs de coordonner leurs actions »(Callon, 1998, dans Dubuisson-Quellier et Lamine, 2008, p. 58, [traduction libre]). Ces différents mécanismes font, par exemple, référence aux marques, aux labels, au prix (Callon et al., 2003, dans Dubuisson-Quellier et Lamine, 2008, p. 58), à la publicité, au merchandising, à la conception du produit ou encore à l'espace de détail disponible (Dubuisson-Quellier, 2006). Ces différents éléments concourent ainsi à la «traduction » du jargon, des procédures et des systèmes organisationnels de la production en des concepts et idées compréhensibles pour le consommateur lambda. Ces éléments lui permettent de faire le lien entre le monde de la production et celui de la consommation.

Dans le contexte du marché, les consommateurs se reposent fortement sur ces mécanismes pour prendre leurs décisions d'achat, comme en témoigne l'importance du champ de recherche du branding ou du commerce de détail. Ce phénomène peut être plus largement désigné sous le terme de "délégation », à travers lequel les consommateurs délèguent aux organisations une variété de fonctions dont ils ne peuvent pas ou ne souhaitent pas s'investir, telles que les opérations de production, de distribution, de sélection ou de contrôle, typiquement l'apanage des entités industrielles (Dubuisson-Quellier, 2006; Dubuisson-Quellier et Lamine, 2008). La notion de « délégation » peut par ailleurs être rapprochée de la notion d'" action collective individualisée » (Micheletti, 2003; Micheletti et al., 2004, dans Dubuisson-Quellier et Lamine, 2008), tels que le " consumérisme politique » ou la « consommation engagée ", à travers lesquels les consommateurs votent ou protestent au moyen de leur chariot de course, mais sans s'engager beaucoup plus substantiellement par ailleurs (Wallner, 2012, p. 95). Plus spécifiquement, le commerce équitable, la consommation verte, ou la consommation locale dénuée d'implication du consommateur dans les processus de production/distribution/promotion peuvent être rapprochés de la notion de "délégation » (Dubuisson-Quellier, 
2006). Le processus des consommateurs en situation de consommation conventionnelle correspond donc à une délégation.

\section{Consommation collaborative et (quasi-)émancipation}

Contrairement à la consommation classique, laquelle peut inclure la consommation éthique (Goodman, 2004; Wallner, 2012), la CC diverge, dans le sens où le consommateur, l'individu, peut intervenir dans la chaîne de création de valeur, soit en amont par la coproduction, la coconception (Ritzer, 2014; Schau et al., 2009), ou l'approvisionnement (Gregson et Crewe, 2003; Belk et al., 1989; Nissanoff, 2006), ce qui a été désigné sous le terme " provision »; soit en aval par l'obtention (Botsman et Rogers, 2010), ce qui a été dénommé "utilisation ». Ces nouveaux consommateurs dits " prosommateurs» (Ritzer, 2014) ou " consomarchands/consommerçants » (Lemaitre et de Barnier, 2015), collaborent avec des entités initialement considérées comme incommensurablement éloignées, telles que les organisations, ou considérées comme non pertinentes telles que les consommateurs, afin de créer des systèmes de circulation de ressources nouveaux. Le rôle classique des intermédiaires conventionnels s'en retrouve bousculé, provoquant la controverse, comme en font foi les critiques et conflits récurrents impliquant les grands joueurs de la CC tels qu'Uber ou Airbnb.

Les consommateurs ne sont plus simplement dans un mode de "délégation ", mais jouissent également d'une "émancipation", puisqu'ils peuvent désormais passer de l'autre côté du miroir et effectuer eux-mêmes de nombreuses tâches, non pas en tant qu'employés formels, mais en tant qu'individus, consommateurs, contractuels, producteurs, ou travailleurs autonomes (Ritzer, 2015; Lambert, 2015; Ertz et al., 2016a, b).

Le concept d'« émancipation »a été développé dans le domaine du commerce équitable et de la consommation locale (Dubuisson-Quellier et Lamine, 2008). D'un côté, les consommateurs peuvent reléguer l'achat éthique ou local à un label, une marque ou tout autre mécanisme utile, ce qui suppose une "délégation ». De l'autre, ils peuvent également s'émanciper de la "délégation» en devenant eux-mêmes des acteurs de provision et de délaissement, notamment à travers les Associations pour le maintien d'une agriculture paysanne (AMAP), en France, en s'impliquant davantage dans le processus de production/distribution/promotion des aliments, en négociant, voire en collaborant directement avec les producteurs (Dubuisson-Quellier et Lamine, 2008).

La CC, en revanche, exclut intrinsèquement la délégation puisqu'elle est contraire à la collaboration. En effet, la $\mathrm{CC}$ correspond à un système de circulation des ressources permettant une permutation du rôle d'utilisateur avec celui de fournisseur, s'accorde mal avec le processus de "délégation ", propre à la consommation conventionnelle. La CC conjugue plutôt, d'une part, l'« émancipation », en permettant des échanges directs entre consommateurs, et d'autre part, ce que nous appellerons la " quasi-émancipation » (Ertz et al., 2016a, b), en intégrant également les mécanismes marchands classiques, lorsque des intermédiaires (p. ex. : plateforme, magasin de seconde main) interviennent dans les échanges de consommateurs à consommateurs et permettent de cimenter ceux-ci en des réseaux d'échange de plus grande ampleur (p. ex. : communautés). Par conséquent, la CC impliquant l'absence d'intermédiaire fait référence à l'« émancipation » alors que la présence d'intermédiaire reflète la configuration des mécanismes de marché classiques et fait ainsi référence à de la "quasi-émancipation", si l'on voit le verre à moitié plein, ou à de la «quasi-délégation », si on voit le verre à moitié vide. On préférera la notion de quasi-émancipation. Le tableau 3 fournit un résumé de ces trois processus du consommateur. 


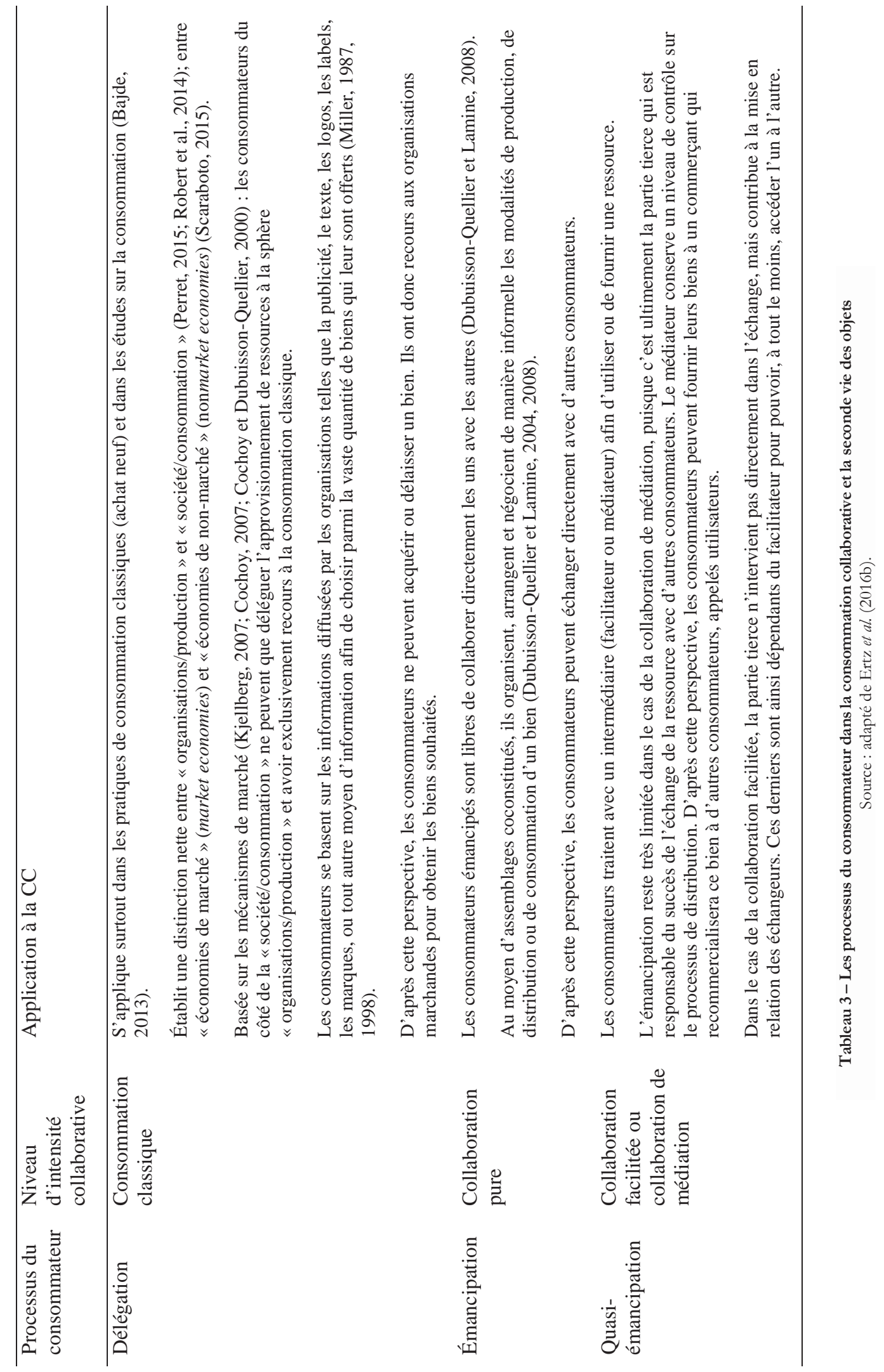


Ces trois processus du consommateur (délégation, émancipation et quasi-émancipation) peuvent être considérés comme un continuum allant de la délégation à l'émancipation et avec la quasi-émancipation au centre. Ce spectre peut ensuite être opposé à un second axe de distance sociale perçue (Sahlins, 1972 dans Arnould et Rose, 2016). Les distances sociales plus courtes impliquent des processus ayant lieu avec la famille, les amis ou les proches, et ensuite avec les connaissances (Price et al., 2000; Curasi et al., 2004); alors que les distances sociales plus larges font référence à de la collaboration avec des étrangers (Lastovicka et Fernandez, 2005).

\section{Conclusion}

Cet article avait principalement pour objectif de présenter une définition de la consommation collaborative ainsi que de proposer des assises conceptuelles sur lesquelles penser la consommation collaborative. Les pratiques de consommation collaboratives sont mouvantes et dynamiques, mais l'apport conceptuel offert dans cet article permet de mieux cerner ces nouveaux modèles d'affaires combinant forces organisationnelles et puissance des réseaux communautaires. Les recherches futures pourraient s'atteler à enrichir, à confirmer empiriquement ou à affiner les éléments proposés dans cet article.

\section{RÉFÉRENCES}

Airgrain, P. (2012). Sharing: Culture and the economy in the Internet age. Amsterdam, Pays-Bas: Amsterdam University Press.

Appadurai, A. (1986). The social life of things: Commodities in cultural perspective. Cambridge, UK: Cambridge University Press.

Arnould, E. J. et Rose, A. S. (2016). Mutuality: Critique and substitute for Belk's "sharing”. Marketing Theory, 16(1), 75-99.

Arsel, Z. et Dobscha, S. (2011). Hybrid pro-social exchange systems: the case of freecycle. Advances in Consumer Research, 39, 66-67.

Bajde, D. (2013). Consumer culture theory (re)visits actor-network theory. Marketing Theory, 13(2), 227-242.

Bardhi, F. et Arnould, E. J. (2005). Thrift shopping: combining utilitarian thrift and hedonic treat benefits. Journal of Consumer Behaviour, 4(4), 223-233.

Bardhi, F. et Eckhardt, G. M. (2012). Access-based consumption: the case of car sharing. Journal of Consumer Research, 39(4), 881-898.

Bazerman, M. H. (2001). Consumer research for consumers. Journal of Consumer Research, 27(4), 499-504.

Belk, R. W. (2014). You are what you can access: Sharing and collaborative consumption online. Journal of Business Research, 67(8), 1595-1600. Doi : 10.1016/j.jbusres.2013.10.001.

Belk, R. W. et Coon, G. S. (1993). Gift giving as agapic love: an alternative to the exchange paradigm based on dating experiences. Journal of Consumer Research, 20(3), 393-417.

Belk, R. W., Sherry, J. F. et Wallendorf, M. (1988). A naturalistic inquiry into buyer and seller behavior at a swap meet. Journal of Consumer Research, 14(4), 449-470.

Botsman, R. (2013). The sharing economy lacks a shared definition. Fast Company. Repéré à https://www.fastcoexist.com/3067443/patagonia-wants-to-refurbish-your-old-clothes-and-sell-them-to-someone-else.

Botsman, R. (2015a). Defining the sharing economy: what is collaborative consumption - and what isn't. Repéré à https://www.fastcoexist.com/3046119/defining-the-sharing-economy-what-is-collaborative-consumption-and-what-isnt.

Botsman, R. (2015b). The sharing economy: dictionary of commonly used terms. Repéré à http://www.collaborativeconsumption.com/2015/11/12/the-sharing-economy-dictionary-of-commonly-used-terms/.

Botsman, R. et Rogers, R. (2010). What's mine is yours. The rise of collaborative consumption. New York, NY: Penguin Press.

Ciari, F., Schuessler, N. et Axhausen, K. W. (2013). Estimation of carsharing demand using an activity-based microsimulation approach: model discussion and some results. International Journal of Sustainable Transportation, 7(1), 70-84. 
Cochoy, F. (2007). A sociology of market-things: on tending the garden of choices in mass retailing. The Sociological Review, 55(2), 109-129.

Cochoy, F. et Dubuisson-Quellier, S. (2000). Introduction : les professionnels du marché - vers une sociologie du travail marchand. Sociologie du Travail, 42(3), 359-368.

Corciolani, M. et Dalli, D. (2014), "Gift-giving, sharing and commodity exchange at Bookcrossing.com : new insights from a qualitative analysis," Management Decision, 52(4), 755-776.

Curasi, C. F., Price, L. L. et Arnould, E. J. (2004), “How individuals' cherished possessions become families' inalienable wealth," Journal of Consumer Research, 31(3), 609-622.

Dalli, D. et Corciolani, M. (2008). Collective forms of resistance: The transformative power of moderate communities. Evidence from the BookCrossing case. International Journal of Market Research, 50(6), 757-775.

Dubuisson-Quellier, S. (2006). Pluralité des formes d'engagement des consommateurs sur les marchés : le cas des produits issus du commerce équitable. Repéré à http://symposcience.lyon.cemagref.fr/exl-doc/colloque/ART-00001962.pdf.

Dubuisson-Quellier, S. et Lamine, C. (2004). Faire le marché autrement : l'abonnement à un panier de fruits et de légumes comme forme d'engagement politique des consommateurs. Sciences de la Société, 144-167. Paris, France : Presses Universitaires du Midi.

Dubuisson-Quellier, S. et Lamine, C. (2008). Consumer involvement in fair trade and local food systems: delegation and empowerment regimes. Geojournal, 73(1), 55-65.

Eckhardt, G. M. et Bardhi, F. (2015). The sharing economy isn't about sharing at all. Harvard Business Review. Repéré à https://hbr.org/2015/01/the-sharing-economy-isnt-about-sharing-at-all.

Ertz, M., et Durif, F. (2017). Définition de la consommation collaboration et des concepts associés. Dans A. Decrop (éd.), La consommation collaborative : Enjeux et défis de la nouvelle société du partage (p. 33-54). Bruxelles : De Boeck.

Ertz, M., Durif, F. et Arcand, M. (2016a). Collaborative consumption or the rise of the two-sided consumer. International Journal of Business and Management, 4(6), 195-209.

Ertz, M., Durif, F. et Arcand, M. (2016b). Collaborative consumption: conceptual snapshot at a buzzword. Journal of Entrepreneurship Education, 19(2), 1-23.

Felson, M. et Spaeth, J. L. (1978). Community structure and collaborative consumption: a routine activity approach. American Behavioral Scientist, 21(4), 614-624.

Fisk, G. (1973). Criteria for a theory of responsible consumption. Journal of Marketing, 37(April), 24-31. Doi : 10.2307/1250047.

Gansky, L. (2010). The mesh: why the future of business is sharing. New York, NY: Penguin Press.

Gielser, M. (2006). Consumer gift systems. Journal of Consumer Research, 33(2), 283-290.

Goodman, M. K. (2004). Reading fair trade: political ecological imaginary and the moral economy of fair trade foods. Political Geography, 23, 891-915.

Gregson, N. et Crewe, L. (2003). Second-hand cultures. Oxford, UK: Berg.

Guiot, D. et Roux, D. (2010). A second-hand shoppers' motivation scale: antecedents, consequences, and implications for retailers. Journal of Retailing, 86(4), 355-371. Doi : 10.1016/j.jretai.2010.08.002.

Hamari, J., Sjöklint, M. et Ukkonen, A. (2015). The sharing economy: why people participate in collaborative consumption. Journal of the Association for Information Science and Technology, 67(9), 2047-2059.

Hawley, A. H. (1950). Human ecology: A theory of community structure. Chicago, MI: The Ronald Press Company.

Herrmann, G. M. et Soiffer, S.M. (1984). For fun and profit. Urban Life, 12(4), 397-421.

John, N. A. (2013). The social logics of sharing. The Communication Review, 16(3), 113-131.

Kaplan, A. M. et Haenlein, M. (2006). Towards a parsimonious definition of traditional and electronic mass customization. Journal of Product Innovation Management, 23(2), 168-182. 
Kijiji (2015, 21 mars). Indice Kijiji de l'économie de seconde main 2015. Repéré à http://consommationresponsable.kijiiji.ca/wpcontent/uploads/2015/01/Indice-Kiijiji.pdf.

Kijiji (2016, 21 mars). Indice Kijiji de l'économie de seconde main 2016. Repéré à http://consommationresponsable.kijiii.ca/wpcontent/uploads/2016/02/Indice-Kijiii-rapport-2016.pdf.

Kijiji (2017, 21 mars). Indice Kijiji de l'économie de seconde main 2017. Repéré à http://consommationresponsable.kiiiji.ca/wpcontent/uploads/2016/02/Indice-Kijiii-rapport-2016.pdf.

Kjellberg, H. (2007). The death of a salesman? Reconfiguring economic exchange in Swedish post-war food distribution. The Sociological Review, 55(2), 65-91.

Lambert, C. (2015). Shadow work: the unpaid, unseen jobs that fill your day. Berkeley, CA : Counterpoint.

Lamberton, C. P. et Rose, R. L. (2012). When is ours better than mine? A framework for understanding and altering participation in commercial sharing systems. Journal of Marketing, 76(4), 109-125. Doi : http://dx.doi.org/10.1509/jm.10.0368.

Lastovicka, J. L., et Fernandez, K. V. (2005). Three paths to disposition : the movement of meaningful possessions to strangers. Journal of Consumer Research, 31(4), 813-823.

Laville, J.-L. (2008). Travail : La nouvelle question politique. Revue du MAUSS, 18.

Leismann, K., Schmitt, M., Rohn, H. et Baedeker, C. (2013). Collaborative consumption: towards a resource-saving consumption culture. Resources, 2(3), 184-203. Doi : 10.3390/resources2030184.

Lemaitre, N. et De Barnier, V. (2015). Quand le consommateur devient commerçant : motivations, production d'expérience et perspectives. Décisions Marketing, 78(avril-juin), 11-28.

Lessig, L. (2008). REMIX: making art and commerce thrive in the hybrid economy. New York, NY: Penguin Press.

Matzler, K., Veider, V. et Kathan, W. (2015). Adapting to the sharing economy. MIT Sloan Management Review. Repéré à http://sloanreview.mit.edu/article/adapting-to-the-sharing-economy/.

Mont, O. K. (2002). Clarifying the concept of product-service system. Journal of Cleaner Production, 10(3), 237-245.

Mont, O. K. (2004). Product-service systems: Panacea or myth? (Thèse de doctorat, Université de Lund, Suède) Repéré à https://lup.lub.lu.se/search/publication/467248.

Nissanoff, D. (2006). Future Shop: how the new auction culture will revolutionize the way we buy, sell, and get the things we really want. New York, NY: The Penguin Press.

Ostrom, E. et Hess, C. (2007). Private and common property rights. Atelier en théorie politique et analyse politique. University d’Indiana. Repéré à http://surface.syr.edu/cgi/viewcontent.cgi?article $=1024 \&$ context $=$ sul.

Parkes, S. D., Marsden, G., Shaheen, S. A. et Cohen, A. P. (2013). Understanding the diffusion of public bikesharing systems: evidence from Europe and North America. Journal of Transport Geography, 31, 94-103.

Perret, B. (2015). De la propriété à l’usage. Esprit, 7, 30-39.

Prahalad, C. K. et Ramaswamy, V. (2000). Co-opting customer competence. Harvard Business Review. Repéré à http://www.sld.cu/galerias/pdf/sitios/infodir/coopting_customer_competence.pdf.

Price, L. L., Arnould, E. J. et Curasi, C. F. (2000). Older consumers' disposition of special possessions. Journal of Consumer Research, 27(2), 179-201.

Ritzer, G. (2014). Prosumption: Evolution, revolution, or eternal return of the same? Journal of Consumer Culture, 14(1), 3-24.

Ritzer, G. (2015). Prosumer capitalism. The Sociological Quarterly, 56(3), 413-445.

Robert, I., Binninger, A. S. et Ourahmoune, N. (2014). La consommation collaborative, le versant encore équivoque de l'économie de la fonctionnalité. Développement durable et territoires, 5(1), 1-30.

Scaraboto, D. (2015). Selling, sharing, and everything in between: The hybrid economies of collaborative networks. Journal of Consumer Research, 42(1), 152-176. 
Schau, H. J., Muñiz, A. M. et Arnould, E. J. (2009). How brand community practices create value. Journal of Marketing, 73(5), $30-51$.

Schor, J. B. (2015). The sharing economy: reports from stage one. Working paper. Boston College. Repéré à http://www.bc.edu/content/dam/files/schools/cas_sites/sociology/pdf/TheSharingEconomy.pdf.

Sherry, J. F. (1990). A Sociocultural analysis of a midwestern american flea market. Journal of Consumer Research, 17(1), 13-30.

Slee, T. (2016). What's yours is mine: against the sharing economy. New York, NY: OR Books.

Solomon, M. R., Zaichkowksy, J. L. et Polegato, R. (2011). Consumer behaviour (5th Canadian edition). Boston, MA: Pearson.

Stone, J., Horne, S. et Hibbert, S. (1996). Car boot sales: a study of shopping motives in an alternative retail format. International Journal of Retail \& Distribution Management, 24(11), 4-15.

Sundararajan, A. (2016). The sharing economy: the end of employment and the rise of crowd-based capitalism. Cambridge, MA : MIT Press.

Tukker, A. (2004). Eight types of product-service system: eight ways to sustainability? Experiences from SusProNet. Business Strategy and the Environment, 13(4), 246-260.

Tussyadiah, I. P. (2015, dir.). An exploratory study on drivers and deterrents of collaborative consumption in travel. Dans I. P. Tussyadiah (dir.). Information and Communication Technologies in Tourism (p. 817-830). Cham, Suisse: Springer International Publishing.

Van de Walle, I., Hébel, P. et Siounandan, N, (2012). Les secondes vies des objets : Les pratiques d'acquisition et de délaissement des produits de consommation. CRÉDOC, Cabier de Recherche n 290.

Varian, H. R. (2000). Buying, sharing and renting information goods. The Journal of Industrial Economics, 48(4), 473-488.

Wallner, T. (2012). Will self-regulating consumption ethics lead to new business paradigms? Journal of Organisational Transformation \& Social Change, 9(2), 109-125. Doi : 10.1386/jots.9.2.109_1.

Zhu, R., Dholakia, U. M., Chen, X. et Algesheimer, R. (2012). Does online community participation foster risky financial behavior? Journal of Marketing Research, 49(3), 394-407. 